\title{
METHOD OF CHOICE OF MANOEUVRE OF DEVIATION OF SHIP FOR SAFE DIVERGENCE
}

\section{СПОСОБ ВЫБОРА МАНЕВРА УКЛОНЕНИЯ СУДНА ДЛЯ БЕЗОПАСНОГО РАСХОЖДЕНИЯ}

\author{
P.A. Chapchay, $P h D$, professor, M.S. Alekseychuk, $P h D$, professor \\ П.А. Чапчай, к.т.н., профессор, М.С. Алексейчук, к.т.н., профессор, \\ National University «Odessa Maritime Academy», Ukraine \\ Наџиональный университет «Одесская морская академия», Украина
}

\begin{abstract}
For the situations of rapprochement of ship with a target procedure of estimation of no unconcern of rapprochement is offered with the help of region of impermissible parameters of motion of ship. For a case, when speed of ship more small to speed of target, the method of determination of course of deviation of ship is considered for warning of possible collision. Shown realization of the offered procedure with the help of the computer program.

In the case when speed of ship more small to speed of target, procedure of choice of course of deviation of ship is offered for warning of collision with a target, using group of dangerous relative courses and all possible relative courses taking into account the relation of speeds of ship and target.

So as in this case absent synonymous dependence between relative and veritable courses, the great number of dangerous relative courses of deviation is determined at first, the graphic reflection of which is a region on a plane. If a point with the value of relative course of rapprochement is in a region, the situation of rapprochement is dangerous. In this case for the choice of optimum course of deviation it is needed to choose a relative course which answers the border of region, and then to define the proper veritable course of deviation. There are cases, when the scopes of region do not achieve the value of safe relative course of deviation, in such case it is required to change speed of ship, at which the values of safe relative course of deviation are within the limits of possible region

Offered realization of the developed procedure with the help of the computer program, thus the resulted examples of determination of course of deviation of ship at his rapprochement with a target. The operative and simplicity of estimation of no unconcern of rapprochement and choice of course of deviation of ship for warning of collision with a target with the help of region of impermissible parameters of motion of ship is shown. It is marked that it is expedient to use the offered computer variant of realization of the got procedure in the perspective navigation informative systems.

The examples of forming of region of impermissible parameters of motion of ship are resulted for the inflicted situation and determination with its help of estimation of no unconcern of rapprochement of ship with a target, and also choice of
\end{abstract}


course of deviation of ship for warning of collision with a target to the condition of its unchanging speed.

Keywords: safety of navigation, warning of collision of vessels, region of impermissible parameters, estimation of danger of rapprochement, divergence by the change of course.

\section{PEФEPAT}

Для ситуацій зближення судна 3 ціллю запропоновано процедуру оцінки небезпечності зближення з допомогою області недопустимих параметрів руху судна. Для випадку, коли швидкість судна менша швидкості цілі, розглянуто спосіб визначення курсу ухилення судна для попередження можливого зіткнення. Показана реалізація пропонованої процедури 3 допомогою комп’ютерної програми.

В разі коли швидкість судна менша швидкості цілі, запропоновано процедуру вибору курсу ухилення судна для попередження зіткнення з ціллю, використовуючи підмножину небезпечних відносних курсів і всіх можливих відносних курсів з урахуванням відношення швидкостей судна і цілі.

Так як в цьому випадку відсутня однозначна залежність між відносним i істинним курсами, то спочатку визначається множина небезпечних відносних курсів ухилення, графічне відображення якої являється областю на площині. Якщо точка із значенням відносного курсу зближення знаходиться в області, то ситуація зближення $\epsilon$ небезпечною. В цьому разі для вибору оптимального курсу ухилення потрібно вибрати відносний курс, який відповідає межі області, а потім визначити відповідний істинний курс ухилення. Існують випадки, коли межі області не досягають значення безпечного відносного курсу ухилення, в такому випадку належить змінити швидкість судна, при якій значення безпечного відносного курсу ухилення знаходяться в межах допустимої області

Запропонована реалізація розробленої процедури 3 допомогою комп'ютерної програми, причому приведені приклади визначення курсу ухилення судна при його зближені з ціллю. Показані оперативність і простота оцінки небезпечності зближення i вибору курсу ухилення судна для попередження зіткнення з ціллю з допомогою області недопустимих параметрів руху судна. Зазначено, що пропонований комп'ютерний варіант реалізації одержаної процедури доцільно використовувати в перспективних навігаційних інформаційних системах.

Приведено приклади формування області недопустимих параметрів руху судна для завданої ситуації та визначення з іiі допомогою оцінки небезпечності зближення судна 3 ціллю, а також вибору курсу ухилення судна для попередження зіткнення з ціллю умові їі незмінної швидкості.

Ключові слова: безпека судноводіння, попередження зіткнень суден, область неприпустимих параметрів, оцінка небезпеки зближення, розходження зміною курсу. 
Постановка проблемы в общем виде и ее связь с важными научными или практическими задачами

В настоящее время число столкновений судов остается на высоком уровне, что в значительной мере обусловлено несовершенной процедурой выявления ситуации опасного сближения, а также отсутствием простого способа оперативного выбора безопасного маневра расхождения. Маневр расхождения изменением курса судна является предпочтительным при наличии достаточного водного пространства, поэтому следует сформировать способ выбора маневра уклонения судна для безопасного расхождения.

Анализ последних достижений и публикаций, в которых начато решение данной проблемы и выделение нерешенных ранее частей общей проблемы

Задача выбора оптимального маневра расхождения, как отмечается в работах [1-3], очень сложная, так как процесс управления движением судна является многомерным с нелинейными и нестационарными характеристиками, причем задача носит игровой характер.

Понимание содержания автономной судовой системы уклонения от столкновения (Collision avoidance) и её теоретическое обоснование излагается в работе [4]. Совместно с алгоритмом по уклонению от столкновения рассмотрены дополнительно правила уклонения от столкновения COLREG. C учетом факторов, влияющих на процесс уклонения от столкновения, рассматриваются требования к автономной навигации, которые способен оценить человек и осуществить управление судном на удовлетворительном уровне, однако принятые решения являются субъективными и могут быть ошибочными, в результате чего может возникнуть столкновение. Исследования по автоматизации управления судном, как указывается в работе, могут быть представлены в классической или компьютерной категориях. Классическая техника основана на математических моделях и алгоритмах. Программы основаны на использовании искусственного интеллекта используют эволюционные алгоритмы, логику фуззи, экспертные методы, нейросеть и комбинация этих методов - гибридные системы. При теоретическом направлении работа не содержит рекомендаций практическому судовождению.

Анализируемые работы вносят значительный вклад в теорию решения проблемы предупреждения столкновений судов, однако они не содержат рекомендаций по оперативному выбору маневров расхождения.

В последнее время появились исследования по проблеме обеспечения безопасного расхождения судов методами внешнего управления с использованием недопустимых областей курсов или скоростей судов [5]. Предлагаемый подход к решению проблемы предупреждения столкновения судов целесообразно использовать и при локально независимом управлением процессом расхождения [5] с использованием недопустимых областей параметров движения судна, которые могут быть реализованы с помощью компьютера в виде оперативной процедуры выбора маневра расхождения судна. Этому вопросу посвящена настоящая статья. 


\section{Формулировка целей статьи (постановка задачи)}

Целью настоящей статьи является рассмотрение процедуры оценки опасности сближения судна с целью и, в случае необходимости, выбор судном маневра расхождения изменением курса с помощью области недопустимых параметров движения судна.

\section{Изложение основного материала исследования с обоснованием полученных научных результатов}

Как указывается в работе [5], в зависимости от соотношения скоростей судна $\mathrm{V}_{1}$ и цели $\mathrm{V}_{2}$ при изменении курса судна $\mathrm{K}_{1}$ возникает особенность изменения относительного курса $\mathrm{K}_{\mathrm{ot}}$. В случае $\mathrm{V}_{1}>\mathrm{V}_{2}$ величина первой производной $\frac{\partial \mathrm{K}_{\mathrm{ot}}}{\partial \mathrm{K}_{1}}$ положительна для всех значений $\mathrm{K}_{1}$, поэтому при изменении курса $\mathrm{K}_{1}$ относительный курс $\mathrm{K}_{\mathrm{ot}}$ может принимать любые значения от 0 до $2 \pi$. Если же $\mathrm{V}_{1}<\mathrm{V}_{2}$ отображение множества относительных курсов в множество истинных курсов судна не является однозначным, так как первая производная $\frac{\partial \mathrm{K}_{\mathrm{ot}}}{\partial \mathrm{K}_{1}}$ может иметь как положительный, так и отрицательный знаки, т. е. каждому относительному курсу, кроме экстремальных, соответствует два значения истинного курса судна, а при изменении курса $\mathrm{K}_{1}$ судна в диапазоне от 0 до $2 \pi$ относительный курс $\mathrm{K}_{\mathrm{ot}}$ будет принимать значения из диапазона $\left[\mathrm{K}_{\text {otmin }}, \mathrm{K}_{\text {otmax }}\right]$, причем:

$$
\mathrm{K}_{\mathrm{otmin}}=\pi+\mathrm{K}_{2}-\arcsin \frac{\mathrm{V}_{1}}{\mathrm{~V}_{2}} \text { и } \mathrm{K}_{\text {otmax }}=\pi+\mathrm{K}_{2}+\arcsin \frac{\mathrm{V}_{1}}{\mathrm{~V}_{2}} .
$$

$\mathrm{B}$ случае $\mathrm{V}_{1}<\mathrm{V}_{2}$ для выбора маневра расхождения изменением курса предлагается следующая процедура. Подмножество относительных курсов $\mathrm{Mn1}{ }_{\mathrm{ot}}$, при которых сближение судна и цели опасно, ограничено значениями $\mathrm{K}_{\mathrm{ot} *}=\gamma^{(1)}=\alpha-\arcsin \frac{\mathrm{d}_{\mathrm{d}}}{\mathrm{D}}$ и $\mathrm{K}_{\mathrm{ot}}^{*}=\gamma^{(2)}=\alpha+\arcsin \frac{\mathrm{d}_{\mathrm{d}}}{\mathrm{D}}$, т.е. $\mathrm{Mn}_{\mathrm{ot}}=\left[\gamma^{(1)}, \gamma^{(2)}\right]$.

В свою очередь, подмножество всех возможных относительных курсов уклонения при $\mathrm{V}_{1}<\mathrm{V}_{2}$ определяется подмножеством $\mathrm{Mn} 2_{\mathrm{ot}}=\left[\mathrm{K}_{\text {otmin }}, \mathrm{K}_{\mathrm{otmax}}\right]$. Если имеется подмножество относительных курсов $\mathrm{Mn} 3_{\mathrm{ot}}$, которое одновременно удовлетворяет условиям $\mathrm{Mn} 3_{\text {ot }} \in \mathrm{Mn} 2_{\text {оt }}$ и $\mathrm{Mn} 3_{\text {ot }} \notin \mathrm{Mn} 1_{\text {ot }}$, то в качестве относительного курса уклонения $\mathrm{K}_{\text {oty }}$ может быть выбран любой относительный курс подмножества $\mathrm{Mn} 3_{\text {ot }}$, т.е. $\mathrm{K}_{\mathrm{oty}} \in \mathrm{Mn} 3_{\mathrm{ot}}$. По выбранному $\mathrm{K}_{\text {oty }}$ можно найти соответствующий истинный курс судна $\mathrm{K}_{1 \mathrm{y}}[9]$.

Предложенная процедура оценки опасности сближения судна с целью и выбор маневра расхождения уклонением при $\mathrm{V}_{1}<\mathrm{V}_{2}$, была реализована на 
компьютере в виде программы, что обеспечило оперативность и простоту решения поставленной задачи.

Для выбора курса уклонения судна при опасном сближении с целью в случае $\mathrm{V}_{1}<\mathrm{V}_{2}$ рассмотрим следующую ситуацию сближения: $\alpha=106^{\circ}, \mathrm{D}=3,0$ мили, $\mathrm{K}_{1}=45^{\circ}, \mathrm{V}_{1}=15$ узла, $\mathrm{K}_{2}=317^{\circ}, \mathrm{V}_{2}=20$ узлов, $\mathrm{d}_{\mathrm{d}}=1$ миля. Ситуация сближения показана на рис. 1 , значение $\min \mathrm{D}=0,34$ мили.

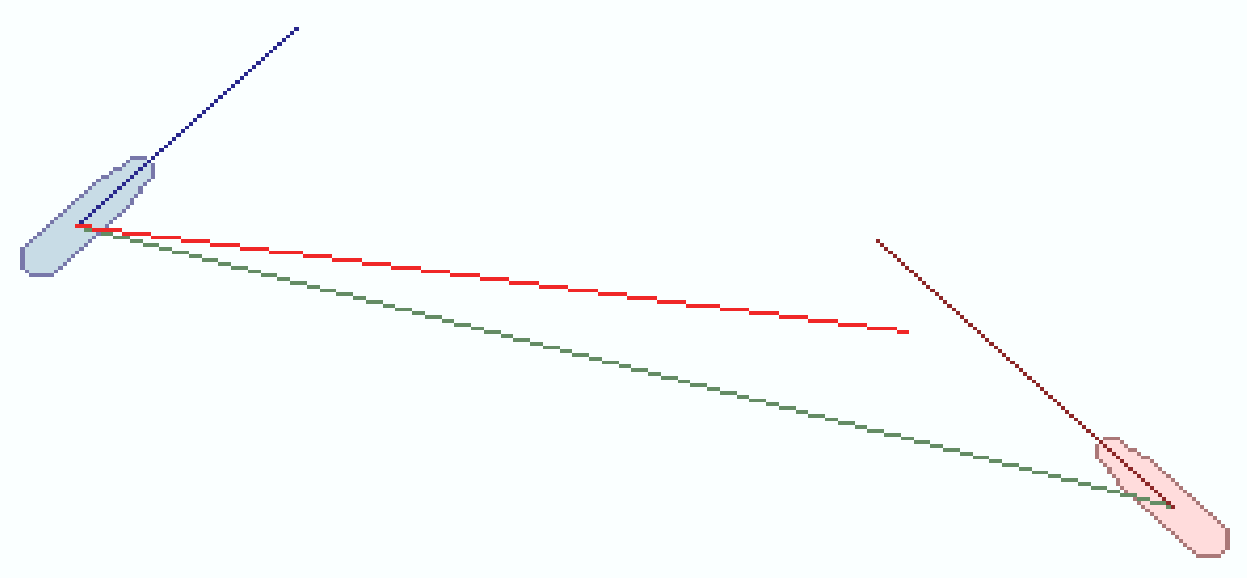

Рис.1. Ситуация сближения при $\mathrm{V}_{1}<\mathrm{V}_{2}$

Графические представления подмножества опасных относительных курсов Mn1 ot и подмножества всех возможных относительных курсов уклонения $\mathrm{Mn} 2_{\text {ot }}$ в системе координат относительных курсов и скоростей судна, полученные с помощью компьютерной программы, показаны на рис. 2. Границы подмножества $\mathrm{Mn} 1_{\text {ot }}$ не зависят от скорости судна и равны $\mathrm{K}_{\mathrm{ot}^{*}}=87^{\circ}$ и $\mathrm{K}_{\mathrm{ot}}^{*}=125^{\circ}$, на рис. 2 они представлены горизонтальными параллельными линиями. Подмножество $\mathrm{Mn} 2$ ot ограничено экстремальными относительными курсами $\mathrm{K}_{\text {otmin }}$ и $\mathrm{K}_{\text {otmax }}$, которые изменяются в зависимости от величины $\mathrm{V}_{1} / \mathrm{V}_{2}$, достигая при $\mathrm{V}_{1}=15$ узлов значений $\mathrm{K}_{\text {otmin }}=89^{\circ}$ и $\mathrm{K}_{\text {otmax }}=185^{\circ}$. Точка с начальным относительным курсом $\mathrm{K}_{\text {oto }}=99^{\circ}$ и скоростью судна $\mathrm{V}_{1}=15$ узлов принадлежит $\mathrm{Mn} 1_{\text {ot }}$. 


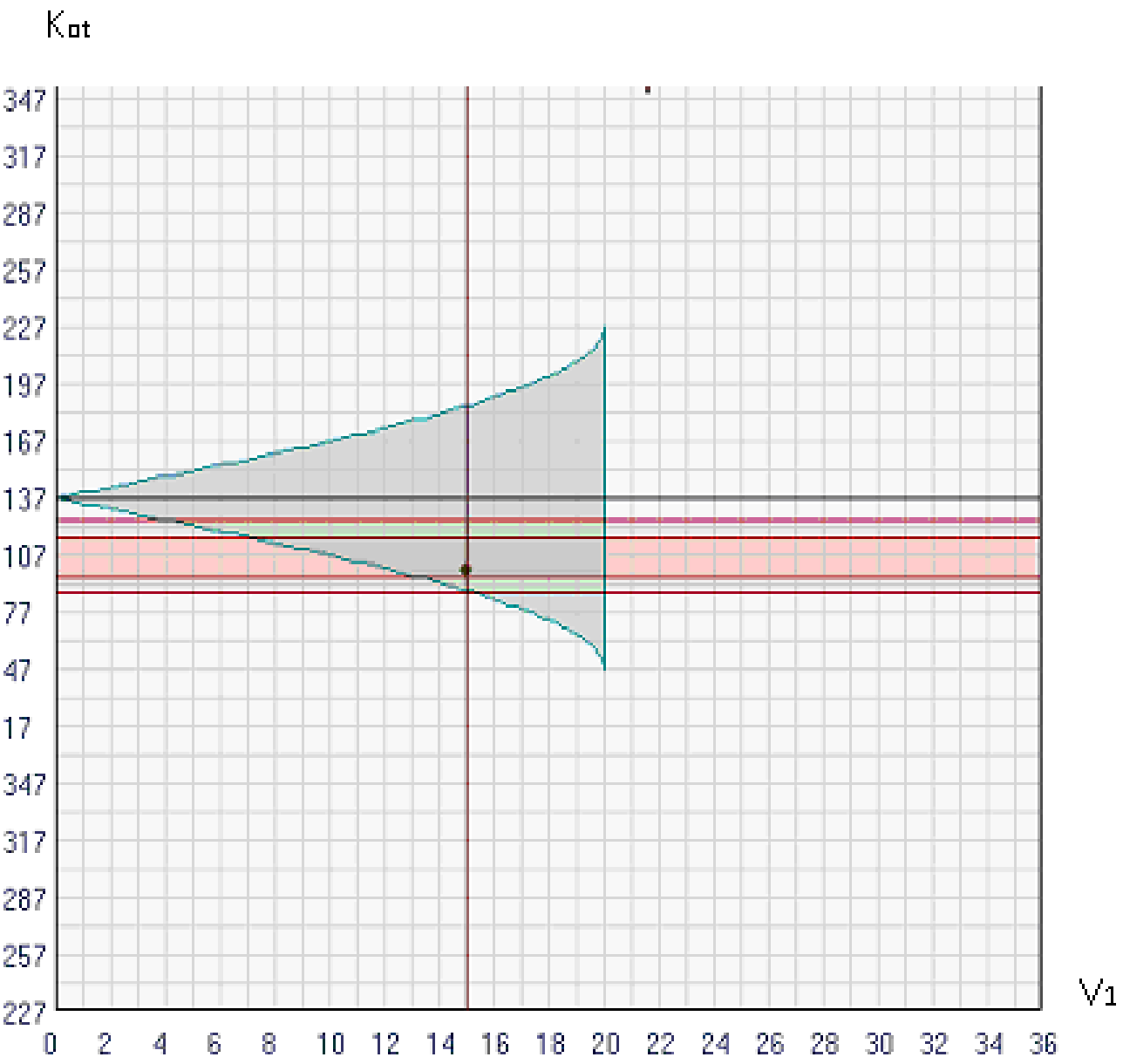

Рис.2. Оџенка опасности начальной ситуащии сближения при $\mathrm{V}_{1}<\mathrm{V}_{2}$

Так как $\mathrm{K}_{\mathrm{ot}^{*}}<\mathrm{K}_{\text {otmin }}$, то уклонением судна влево невозможно увеличить $\min \mathrm{D}$ до значения $\mathrm{d}_{\mathrm{d}}$. Поэтому вводом значения курса уклонения судна $\mathrm{K}_{1 \mathrm{y}}$ поворотом вправо с помощью линейки прокрутки изменяем величину относительного курса $\mathrm{K}_{\text {oty }}$ и положение горизонтальной линии, соответствующей ему, до тех пор пока не совместим ее с верхней границей $\mathrm{K}_{\mathrm{ot}}^{*}$ подмножества $\mathrm{Mn}_{\mathrm{ot}}$, как показано на рис. 3. Из рисунка видно, что $\mathrm{K}_{\mathrm{oty}}=\mathrm{K}_{\mathrm{ot}}^{*}=125^{\circ}$. При этом вводимое значение курса уклонения судна составило $\mathrm{K}_{1 \mathrm{y}}=109^{\circ}$, а значение $\min \mathrm{D}=0,99$ мили.

Таким образом, в случае $\mathrm{V}_{1}<\mathrm{V}_{2}$ процедура оценки опасности сближения судна с целью и выбора курса уклонения судна, реализованная в компьютерной программе, позволяет оперативно визуально оценить опасность ситуации сближения и в случае необходимости с помощью линейки прокрутки элементарно определить курс уклонения, при котором $\min \mathrm{D}=\mathrm{d}_{\mathrm{d}}$. 


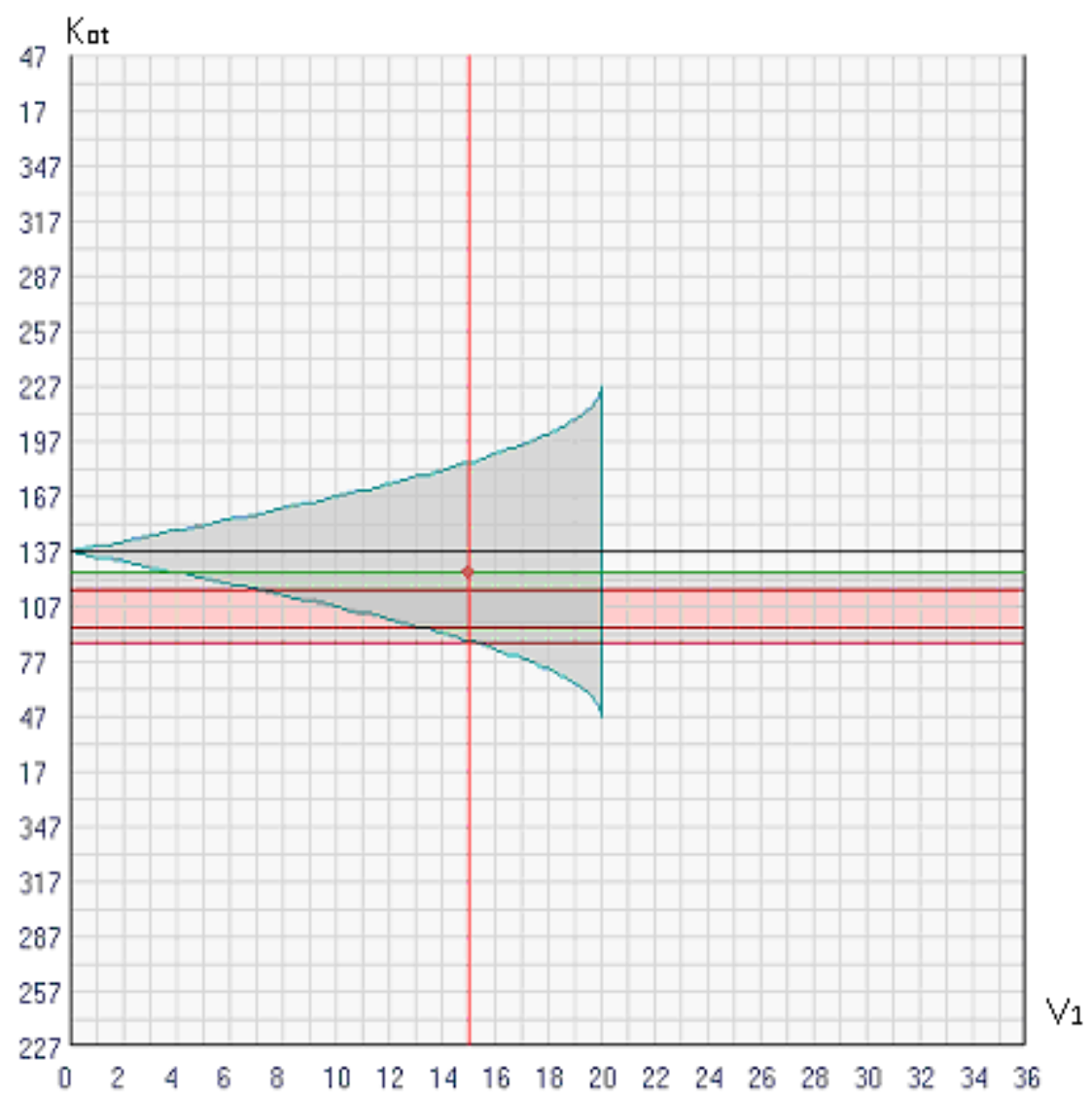

Рис. 3. Выбор курса уклонения судна $\mathrm{K}_{1 \mathrm{y}}$ при $\mathrm{V}_{1}<\mathrm{V}_{2}$

\section{Выводы и перспектива дальнейшей работы по данному направлению}

Для ситуации, когда скорость судна меньше скорости цели, предложена процедура выбора курса уклонения судна для предупреждения столкновения с целью, используя подмножества опасных относительных курсов и всех возможных относительных курсов с учетом отношения скоростей судна и цели, которая также содержит научную новизну в тематике предупреждения столкновения судов. Предложена реализация предложенной процедуры с помощью компьютерной программы и приведены примеры определения курса уклонения судна при его сближении с целью. Обоснована оперативность и простота оценки опасности сближения и выбор маневра уклонения судна. Предложенный компьютерный вариант реализации рассмотренной процедуры целесообразно использовать в перспективных навигационных информационных системах.

\section{ЛИТЕРАТУРА}

1. Lisowski J. Game and computational intelligence decision making algorithms for avoiding collision at sea/ Lisowski J. // Proc. of the IEEE Int. Conf. on Technologies for Homeland Security and Safety. - 2005. - Gdańsk. - pp. 71-78.

2. Lisowski J. Game control methods in navigator decision support system/ Lisowski J. // The Archives of Transport. - 2005. - No 3-4, Vol. XVII. - 
pp. 133-147.

3. Lisowski J. Dynamic games methods in navigator decision support system for safety navigation/ Lisowski J. // Advances in Safety and Reliability. - 2005. Vol. 2. - London-Singapore, Balkema Publishers. - pp. 1285-1292.

4. Statheros Thomas. Autonomous ship collision avoidance navigation concepts, technologies and techniques / Statheros Thomas, Howells Gareth, McDonaldMaier Klaus. // J. Navig. 2008. 61, № 1, pp. 129-142.

5. Бурмака И.А. Управление судами в ситуации опасного сближения / И.А Бурмака., Э.Н Пятаков., А.Ю. Булгаков - LAP LAMBERT Academic Publishing, - Саарбрюккен (Германия), -2016. - 585 с. 